\title{
SUPERLACUNARY CUSP FORMS
}

\author{
KEN ONO AND SINAI ROBINS
}

(Communicated by Dennis A. Hejhal)

Dedicated to Basil Gordon on the occasion of his retirement

\begin{abstract}
Many researchers have studied Euler product identities of weight $k=\frac{1}{2}$ and $k=\frac{3}{2}$, often related to the Jacobi Triple Product identity and the Quintuple Product identity. These identities correspond to theta series of weight $k=\frac{1}{2}$ and $k=\frac{3}{2}$, and they exhibit a behavior which is defined as superlacunary. We show there are no eigen-cusp forms of integral weight which are superlacunary. For half-integral weight forms with $k \geq \frac{5}{2}$, we give a mild condition under which there are no superlacunary eigen-cusp forms. These results suggest the nonexistence of similar Euler-Product identities that arise from eigen-cusp forms with weight $k \neq \frac{1}{2}$ or $\frac{3}{2}$.
\end{abstract}

\section{INTRODUCTION}

The Dedekind $\eta$-function is defined by

$$
\eta(\tau)=x^{\frac{1}{24}} \prod_{n=1}^{\infty}\left(1-x^{n}\right),
$$

where $\tau$ lies in the upper half plane $\mathscr{H}=\{\tau \mid \operatorname{Im}(\tau)>0\}$ and $x=e^{2 \pi i \tau}$. We maintain this notation throughout the paper. The Dedekind $\eta$-function is a modular form of weight $\frac{1}{2}$ on the subgroup $\Gamma_{0}\left(24^{2}\right)$ of $\Gamma=S L_{2}(\mathbb{Z})$, with a multiplier system. An $\eta$-product is defined to be any function $f(\tau)$ of the form

$$
f(\tau)=\prod_{\delta \mid N} \eta(\delta \tau)^{r_{\delta}},
$$

where $r_{\delta} \in \mathbb{Z}$. This is a modular form of weight $k=\frac{1}{2} \sum_{\delta \mid N} r_{\delta}$ with a multiplier system [9], [16]. An $\eta$-polynomial is defined to be any finite linear combination of $\eta$-products. The study of the Fourier coefficients of $\eta$-products and $\eta$-polynomials are related to many well-known number-theoretic functions, including partition functions and quadratic form representation numbers. They also arise from representations of the "monster" group [3] and the Mathieu group $M_{24}$ [15]. The $\eta$-products are building blocks of other modular forms in

Received by the editors June 25, 1993.

1991 Mathematics Subject Classification. Primary 11F11, 11F12, 11F37; Secondary 11P81.

Key words and phrases. Modular forms, lacunarity, superlacunarity. 
the sense that all forms on the full modular group $\Gamma$ are linear combinations of $\eta$-products [9]. To facilitate the subject with examples, we recall Euler's identity

$$
\eta(\tau)=x^{\frac{1}{24}} \sum_{n=-\infty}^{\infty}(-1)^{n} x^{\frac{3 n^{2}+n}{2}}
$$

Jacobi later obtained

$$
\eta^{3}(\tau)=x^{\frac{1}{8}} \sum_{n=0}^{\infty}(-1)^{n}(2 n+1) x^{\frac{n^{2}+n}{2}} .
$$

Moreover, the classical theta function is defined by

$$
\theta(x)=\sum_{-\infty}^{\infty} x^{n^{2}}
$$

and has the $\eta$-product

$$
\theta(-x)=\eta^{2}(\tau) \eta^{-1}(2 \tau) .
$$

When one applies the canonical Fricke involution $\tau \rightarrow-1 / 2 \tau$ to the Riemann surface $X_{0}(2)$, one obtains the classical identity of Gauss:

$$
\eta^{-1}(\tau) \eta^{2}(2 \tau)=x^{\frac{1}{8}} \sum_{n=-\infty}^{\infty} x^{\frac{n^{2}+n}{2}}
$$

These are all examples of a more general phenomenon-the Jacobi TripleProduct identity [1]:

$$
\prod_{n=1}^{\infty}\left(1-x^{2 n}\right)\left(1+z x^{2 n-1}\right)\left(1+z^{-1} x^{2 n-1}\right)=\sum_{n=-\infty}^{\infty} x^{n^{2}} z^{n} .
$$

There are other identities of weight $\frac{3}{2}$ of a similar flavor:

$$
\begin{aligned}
\eta^{5}(\tau) \eta^{-2}(2 \tau) & =x^{\frac{1}{24}} \sum_{n=-\infty}^{\infty}(6 n+1) x^{\frac{3 n^{2}+n}{2}}, \\
\eta^{-2}(\tau) \eta^{5}(2 \tau) & =x^{\frac{1}{3}} \sum_{n=-\infty}^{\infty}(-1)^{n}(3 n+1) x^{3 n^{2}+2 n}, \\
\eta^{2}(\tau) \eta^{-1}(2 \tau) \eta^{2}(4 \tau) & =x^{\frac{1}{3}} \sum_{n=-\infty}^{\infty}(3 n+1) x^{3 n^{2}+2 n} .
\end{aligned}
$$

These follow from the Quintuple-Product identity [6], [28]:

$$
\begin{aligned}
& \prod_{n=1}^{\infty}\left(1-x^{2 n}\right)\left(1-z x^{2 n-1}\right)\left(1-z^{-1} x^{2 n-1}\right)\left(1-z^{2} x^{4 n-4}\right)\left(1-z^{-2} x^{4 n-4}\right)= \\
& =\sum_{n=-\infty}^{\infty} x^{3 n^{2}-2 n}\left(z^{3 n}+z^{-3 n}-z^{3 n-2}-z^{-3 n+2}\right) .
\end{aligned}
$$

All of the above $\eta$-product identities also come from McDonald's Identities on affine Lie Algebras [14]. They are all weight $\frac{1}{2}$ and weight $\frac{3}{2}$ modular forms 
on congruence subgroups of $\Gamma$. Moreover, they can all be realized as Shimura's [26] theta functions

$$
\theta(\psi ; \tau)=\sum_{n=-\infty}^{\infty} \psi(n) n^{\nu} x^{n^{2}}
$$

where $\psi$ is a primitive character, $\psi(-1)=(-1)^{\nu}$, and $\nu=0$ corresponds to weight $\frac{1}{2}$ forms while $\nu=1$ corresponds to weight $\frac{3}{2}$ forms. To study this phenomenon, we first recall the definition of lacunarity:

Definition. A power series is called lacunary if the arithmetic density of its nonzero coefficients is zero. More precisely, the series $x^{\nu} \sum_{n=0}^{\infty} c(n) x^{n}$ is lacunary if

$$
\lim _{t \rightarrow \infty} \frac{\operatorname{card}\{n \mid n \leq t \text { and } c(n) \neq 0\}}{t}=0 .
$$

Many lacunary forms play important roles in the theory of elliptic curves. For example, the lacunary form $\eta^{2}(4 \tau) \eta^{2}(8 \tau)$ is the inverse Mellin transform of the Hasse-Weil $L$-function of the elliptic curve $y^{2}=x^{3}-x$. It is the image under the Shimura map of the forms of weight $\frac{3}{2}$ which arise in Tunnell's work on the congruent number problem (see [12] and [30]).

Serre [24] has determined all the even integers $r$ for which $\eta(\tau)^{r}$ is lacunary. The result is as follows:

Theorem 1 (Serre). Suppose $r>0$ is even. Then $\eta(\tau)^{r}$ is lacunary if and only if $r=2,4,6,8,10,14$, or 26 .

Serre shows the connections between the density of the nonzero Fourier coefficients of $f(\tau)$, an integral weight modular form, and the representablity of $f(\tau)$ as a linear combination of Hecke character forms [18], [24]. Similar methods have been used [8] to show there are only finitely many lacunary forms $\eta^{r}(\tau) \eta^{s}(2 \tau)$ for all $r+s$ even, and they are all listed.

Serre asks [24] if there are any other odd values of $r$ for which $\eta(\tau)^{r}$ is lacunary. We will give evidence supporting Serre's conjecture. The following definition, due to Gordon, captures an essential property of all of the above identities:

Definition. A power series is called superlacunary if it has the form

$$
f(x)=\sum_{n=-\infty}^{\infty} d\left(a n^{2}+b n+c\right) x^{a n^{2}+b n+c},
$$

where $a>0$ and $a, b, c \in \mathbb{Z}$.

We lose no generality by assuming that $a, b, c$ are integers since we can always replace $x$ by a power of $x$ to clear the denominators. More generally, we may define any finite linear combination of such forms to be superlacunary. Because of the Serre-Stark basis theorem for modular forms of weight $\frac{1}{2}$ [25], it follows that any weight $\frac{1}{2}$ modular form on a congruence subgroup of $\Gamma$ is a linear combination of 'twisted' theta series and is hence a superlacunary form.

We note that the product of two superlacunary series is lacunary by an easy extension of Landau's theorem on quadratic forms [13], [21]. Examples (2) 
and (3) show that $\eta(\tau)$ and $\eta^{3}(\tau)$ are both superlacunary. Hence if $\eta^{a}(\tau)$ is superlacunary, then $\eta^{a+1}(\tau)$ and $\eta^{a+3}(\tau)$ are both lacunary. By Serre, the only possible values of $a$ are $a=1,3,5$, and 7 . It is easy to verify that $\eta^{5}(\tau)$ and $\eta^{7}(\tau)$ both are not superlacunary. Consequently, the only superlacunary powers of the Dedekind $\eta$-function are $\eta(\tau)$ and $\eta^{3}(\tau)$. We will show that it is difficult for a superlacunary eigen-cusp form to have weight $k \geq \frac{5}{2}$. As corollaries to our main theorems we obtain

Corollary 1. There are no superlacunary integer weight cuspidal Dedekind $\eta$ polynomials which are eigenforms of the Hecke operators $T_{p}$.

Corollary 2. Let $k \in \frac{1}{2}+\mathbb{Z}$, and let $f(\tau)$ be a superlacunary Dedekind $\eta$ polynomial which is a cuspidal eigenform of the Hecke operators $T_{p^{2}}$. Let

$$
f(\tau)=\sum_{n=-\infty}^{\infty} d\left(a n^{2}+b n+c\right) x^{a n^{2}+b n+c}
$$

be its Fourier expansion at infinity. If $b^{2}-4 a c \neq 0$, then $k=\frac{1}{2}$ or $k=\frac{3}{2}$.

\section{GALOIS REPRESENTATIONS AND SHIMURA'S LIFT}

Our theorems are a consequence of the theory of Galois representations associated to modular forms [4] and the Shimura lift for half-integral weight cusp forms [2], [26]. These theorems were turning points of research in the 70s and we quote them below:

Theorem (Deligne-Serre). Let $f$ be a modular form of type $(k, \epsilon)$ on $\Gamma_{0}(N)$ with $\epsilon$ a real character satisfying the following two conditions:

(a) $f$ is an eigenform of the Hecke operators $T_{p}$ for all primes $p \in \mathbb{Z}$ with $p \nmid N$.

(b) The Fourier coefficients $a(n)$ of $f$ are rational integers, and $a(1)=1$.

If $k \geq 1$, then for every rational prime $l$, there is a continuous linear representation

$$
\rho_{l}: \operatorname{Gal}(\mathbb{Q} / \mathbb{Q}) \rightarrow G L_{2}\left(\mathbb{Z}_{l}\right)
$$

such that for all primes $p$ with $p \nmid l N$ we have:

$$
\begin{aligned}
\operatorname{tr}\left(\rho_{l}(\operatorname{Frob}(p))\right. & \equiv a(p) \bmod (l), \\
\operatorname{det}\left(\rho_{l}(\operatorname{Frob}(p))\right. & \equiv \epsilon(p) p^{k-1} \bmod (l) .
\end{aligned}
$$

Given a modular form $f$ and its associated representation $\rho_{l}$, congruences on the Fourier coefficients $a(n)$ hold when the image $\rho_{l}(\mathrm{Gal}(\mathbb{Q} / \mathbb{Q}))$ does not contain $S L_{2}\left(\mathbb{Z}_{l}\right)$. For $l>3$, this happens only if $\tilde{\rho}_{l}(\mathrm{Gal}(\mathbb{Q} / \mathbb{Q}))$ does not contain $S L_{2}\left(F_{l}\right)$, where $\tilde{\rho}_{l}$ is the reduction of $\rho_{l}$ modulo $l$. Such primes $l$ are called exceptional. These ideas go back to Serre and Swinnerton-Dyer [29]. Moreover, it is known that only forms of weight 1 or of CM-type have infinitely many exceptional primes [17], [19].

Here we show that $\epsilon^{-1}(p) p^{1-k} a^{2}(p)$ controls the image of $\tilde{\rho}_{l}$ in $P G L_{2}\left(F_{l}\right)$. For example, assume that $\sigma=\tilde{\rho}_{l}(\operatorname{Frob}(p))$ has order 2 in $P G L_{2}\left(F_{l}\right)$. Hence the eigenvalues $\alpha$ and $\beta$ of $\sigma$ satisfy $\alpha=-\beta$. Consequently we get

$$
\epsilon^{-1}(p) p^{1-k} a^{2}(p) \equiv \frac{\operatorname{tr}^{2}\left(\tilde{\rho}_{l}(\operatorname{Frob}(p))\right)}{\operatorname{det}\left(\tilde{\rho}_{l}(\operatorname{Frob}(p))\right)} \equiv \frac{(\alpha-\alpha)^{2}}{-\alpha^{2}} \equiv 0 \bmod (l) .
$$


Similarly, if $\sigma=\tilde{\rho}_{l}(\operatorname{Frob}(p))$ has order 1 in $P G L_{2}\left(F_{l}\right)$, then

$$
\epsilon^{-1}(p) p^{1-k} a^{2}(p) \equiv 4 \bmod (l) .
$$

We now state a special case Shimura's fundamental theorem defining a correspondence between half-integral weight modular forms and integral weight modular forms [26].

Theorem (Shimura). Let $\chi$ be a Dirichlet character mod $4 N, \lambda$ a positive integer, and $t$ a positive square-free positive integer. Let

$$
F(\tau)=\sum_{n=1}^{\infty} d(n) x^{n} \in S_{\lambda+\frac{1}{2}}(4 N, \chi)
$$

be an eigenform of the Hecke operators $T_{p^{2}}$ with eigenvalues $\lambda_{p}$. Define $A_{t}(n)$ by the following identity:

$$
\begin{aligned}
\sum_{n=1}^{\infty} \frac{A_{t}(n)}{n^{s}} & =L\left(s-\lambda+1, \chi_{t}^{(\lambda)}\right) \sum_{m=1}^{\infty} \frac{d\left(t m^{2}\right)}{m^{s}} \\
& =d(t) \prod_{p} \frac{1}{1-\lambda_{p} p^{-s}+\chi^{2}(p) p^{2 \lambda-1-2 s}},
\end{aligned}
$$

where $\chi_{t}^{(\lambda)}(m)=\chi(m)\left(\frac{-1}{m}\right)^{\lambda}\left(\frac{t}{m}\right)$ is a Dirichlet character mod $4 N t$. Define the normalized image under the Shimura map by

$$
S_{t}(F)=\frac{1}{d(t)} \sum_{n=1}^{\infty} A_{t}(n) x^{n} .
$$

Then for $\lambda>1, S_{t}(F) \in S_{2 \lambda}\left(2 N, \chi^{2}\right)$, and for $\lambda=1, S_{t}(F) \in M_{2 \lambda}\left(2 N, \chi^{2}\right)$. Note that by construction $S_{t}(F)$ is a normalized eigenform of the integral weight Hecke operators $T_{p}$ with eigenvalues $\lambda_{p}$.

By Shimura [26, p. 459] there exists at least one square-free $t$ for which $d(t) \neq 0$. Note that the lift takes half-integral weight cusp forms to integral weight cusp forms when $\lambda>1$. When $\lambda=1$, the image $S_{t}(F)$ is not necessarily a cusp form. In fact, it is for this reason that superlacunary eigenform identities exist for weight $\frac{3}{2}$. Specifically, each of the weight $\frac{3}{2}$ identities corresponds to an Eisenstein series under the Shimura lift. In this vein, we note that Sturm [27] has proved that any weight $\frac{3}{2}$ cusp form which lifts to a weight 2 Eisenstein series must be a linear combination of theta series of type (11). Moreover, the results of the next section suggest that new $\eta$-product identities of superlacunary type, if any, arise in this manner.

\section{THE MAIN THEOREMS}

Although the theorems below hold in more generality, we shall assume that our integral weight modular forms have integer Fourier coefficients and real Nebentypus character. It should be clear from context whether we use the term eigenform to denote integral weight or half-integral weight forms. 
Theorem 1. There are no superlacunary integer weight cusp forms which are eigenforms of the Hecke operators $T_{p}$.

Proof. Suppose $k$ is the weight of the superlacunary eigen-cusp form

$$
f(\tau)=\sum_{n=1}^{\infty} d(n) x^{n} .
$$

By the Chebotarev density theorem, for all primes $l$, there must be a positive density of primes $p$ such that

$$
\epsilon^{-1}(p) p^{1-k} d^{2}(p) \equiv 4 \bmod (l) .
$$

Again, these primes correspond to the identity element of the image of the Galois representation in $P G L_{2}\left(F_{l}\right)$. However, the density of primes represented by any quadratic polynomial in one variable is zero. The number of primes $p<N$ satisfying $p=a n^{2}+b n+c$ is $O(\sqrt{N})$. Moreover, $\pi(N)$ is asymptotically equal to $\frac{N}{\log N}$. Since the exponents of a superlacunary form are given by such a quadratic, the theorem follows.

Because of this theorem we may now assume that a superlacunary eigen-cusp form has half-integral weight.

Theorem 2. Let $F \in S_{\lambda+\frac{1}{2}}(4 N, \chi)$ with $\lambda$ an integer be an eigen-cusp form of the Hecke operators $T_{p^{2}}$. Let

$$
F(\tau)=\sum d\left(a n^{2}+b n+c\right) x^{a n^{2}+b n+c}
$$

be superlacunary. If $b^{2}-4 a c \neq 0$, then $\lambda=0$ or 1 .

Proof. Let $F(\tau)=\sum d\left(a n^{2}+b n+c\right) x^{a n^{2}+b n+c}$ be an eigen-cusp form of all the Hecke operators $T_{p^{2}}$ of half-integral weight with $\lambda>1$. This condition forces the image to be a cusp form. Note the following argument still holds when $\lambda=1$ and $S_{t}(F)$ is a cusp form. By Shimura's theorem, $f(\tau)=S_{t}(F)=\sum_{n=1}^{\infty} a(n) x^{n}$ is a normalized eigen-cusp form of the Hecke operators $T_{p}$. As mentioned earlier, we may choose a positive, square-free integer $t$ such that $d(t) \neq 0$.

For any prime $p$, the Shimura lift gives us

$$
a(p)=\chi_{t}^{(\lambda)}(p) p^{\lambda-1} d(t)+d\left(t p^{2}\right) .
$$

We will show that $d\left(t p^{2}\right)=0$ for a set of primes with density 1 .

We are interested in the set of primes $p$ that satisfy

$$
t p^{2}=a n^{2}+b n+c .
$$

By completing the square, we reduce this to the Pell equation [20]

$$
x^{2}-D y^{2}=b^{2}-4 a c,
$$

where $x=2 a n+b, y=2 p$, and $D=a t$. If $D$ is a perfect square, then $b^{2}-4 a c \neq 0$ assures at most finitely many prime factors of $x^{2}-D y^{2}$, since the positive integer $x+\sqrt{D} y$ can now only take on finitely many values. Hence there are at most finitely many primes $p$ with $d\left(t p^{2}\right) \neq 0$ and we have $d\left(t p^{2}\right)=0$ for almost all $p$.

If $D$ is not a perfect square, then it is well known that the solutions of this equation are given by

$$
\left(x_{0}+\sqrt{D} y_{0}\right)^{k}
$$


by Dirichlet's unit theorem in $\mathbb{Q}(\sqrt{D})$. It is evident that the solutions $(x, y)$ grow exponentially with $k$. Therefore the set of primes $p$ such that $y=2 p$ has density 0 . Consequently we again have $d\left(t p^{2}\right)=0$ for almost all primes $p$.

Hence

$$
a(p)=\chi_{t}^{(\lambda)}(p) p^{\lambda-1} d(t)
$$

for almost all primes $p$. It follows that for each prime $l$ not dividing $d(t)$, the set of primes $p$ satisfying

$$
\chi^{-2}(p) p^{1-2 \lambda} a^{2}(p) \not \equiv 0 \bmod (l)
$$

has density 1. By the Chebotarev density theorem, this means that the Galois representation $\tilde{\rho}_{l}$ cannot contain $S L_{2}\left(F_{l}\right)$ because the order 2 elements in $P S L_{2}\left(F_{l}\right)$ correspond to

$$
a(p) \equiv 0 \quad \bmod (l) .
$$

However, it is clear that $S L_{2}\left(F_{l}\right)$ contains

$$
\left(\begin{array}{ccc}
0 & 1 & \\
& -1 & 0
\end{array}\right) \text {. }
$$

This implies that all rational primes $l$ are exceptional for our form $f(\tau)$. By [18], this forces $f$ to be a CM-form. It is well known that the set of primes $p$ with $a(p)=0$ has positive density for CM-forms. However, by (12) the set of primes $p$ where $a(p)=0$ has density 0 . Consequently, $F$ is not a half-integral weight form.

\section{DiscusSION AND EXAMPLES}

As noted earlier, the product of any two superlacunary series is lacunary. We can therefore construct infinite families of lacunary forms of weight 1,2 , and 3 . For example, we list the weight 3 lacunary families from the $\eta$-products (3), (7), (8), and (9) given in $\S 1$ :

$$
\begin{aligned}
& \eta^{3}(a \tau) \eta^{3}(b \tau) \\
& \eta^{3}(a \tau) \eta^{5}(b \tau) \eta^{-2}(2 b \tau) \\
& \eta^{3}(a \tau) \eta^{-2}(b \tau) \eta^{5}(2 b \tau) \\
& \eta^{3}(a \tau) \eta^{2}(b \tau) \eta^{-1}(2 b \tau) \eta^{2}(4 b \tau) \\
& \eta^{5}(a \tau) \eta^{5}(b \tau) \eta^{-2}(2 a \tau) \eta^{-2}(2 b \tau) \\
& \eta^{5}(a \tau) \eta^{5}(2 b \tau) \eta^{-2}(a \tau) \eta^{-2}(2 b \tau) \\
& \eta^{5}(a \tau) \eta^{-2}(2 a \tau) \eta^{2}(b \tau) \eta^{-1}(2 b \tau) \eta^{2}(4 b \tau) \\
& \eta^{-2}(a \tau) \eta^{-2}(b \tau) \eta^{5}(2 a \tau) \eta^{5}(2 b \tau) \\
& \eta^{-2}(a \tau) \eta^{5}(2 a \tau) \eta^{2}(b \tau) \eta^{-1}(2 b \tau) \eta^{2}(4 b \tau) \\
& \eta^{2}(a \tau) \eta^{-1}(2 a \tau) \eta^{2}(4 a \tau) \eta^{2}(b \tau) \eta^{-1}(2 b \tau) \eta^{2}(4 b \tau)
\end{aligned}
$$

Note that these forms can be explicitly written as linear combinations of Hecke character forms for all integers $a$, and $b$, by Ribet and Serre [19], [24].

The authors ask the following three questions, the second two of which are due to Basil Gordon: 
(1) Are there any superlacunary forms of weight other than $\frac{1}{2}$ or $\frac{3}{2}$ ? In particular, is there some integer-valued function $d(n)$ for which $\sum_{n=1}^{\infty} d(n) x^{n^{2}}$ is an eigen-cusp form of weight $k>\frac{3}{2}$ ?

(2) Is a half-integral weight modular form lacunary if and only if it is superlacunary?

(3) If the cardinality of the set $\{a(n) \neq 0 \mid 0 \leq n \leq N\}$ of Fourier coefficients of an eigen-cusp form is $O\left(N^{1-\epsilon}\right)$, is the form necessarily superlacunary? This would mean that there are no forms 'between' a lacunary and a superlacunary form.

\section{REFERENCES}

1. G. Andrews, The theory of partitions, Addison-Wesley, Reading, MA, 1976.

2. B. Cipra, On the Shimura lift, apres Selberg, J. Number Theory 32 (1989), 58-64.

3. J. H. Conway and S. P. Norton, Monstrous moonshine, Bull. London Math. Soc. 11 (1979), 308-339.

4. P. Deligne, Formes modulaires et representations l-adiques, Lecture Notes in Math., vol. 179, Springer-Verlag, New York, 1971, pp. 139-172.

5. D. Dummit, H. Kisilevsky, and J. McKay, Multiplicative products of $\eta$-functions, Contemp. Math., vol. 45, Amer. Math. Soc., Providence, RI, 1985, pp. 89-98.

6. B. Gordon, Some identities in combinatorial analysis, Quart. J. Math. Oxford Ser. (2) 12 (1961), 285-290.

7. B. Gordon and K. Hughes, Multiplicative properties of $\eta$-products II, Contemp. Math., vol. 143, Amer. Math. Soc., Providence, RI, 1993, pp. 415-430.

8. B. Gordon and S. Robins, Lacunarity of Dedekind $\eta$-products, preprint.

9. B. Gordon and D. Sinor, Multiplicative properties of $\eta$-products, Lecture Notes in Math., vol. 1395, Springer-Verlag, New York, 1988, pp. 173-200.

10. V. G. Kac, Infinite dimensional algebras, Dedekind's $\eta$-function, classical Möbius function and the Very Strange Formula, Adv. Math. 30 (1978), 85-136.

11. V. G. Kac and D. H. Peterson, Affine Lie algebras and Hecke modular forms, Bull. Amer. Math. Soc. (N.S.) 3 (1980), 1057-1061.

12. N. Koblitz, Introduction to elliptic curves and modular forms, Springer-Verlag, New York, 1984.

13. E. Landau, Über die Einteilung der positiven ganzen Zahlen in vier Klassen nach der Mindestzahl der zu ihrer additiven Zusammensetzung erforderlichen Quadrate, Arch. Math. Phys. (3) 13 (1908), 30-312.

14. I. G. MacDonald, Affine root systems and Dedekind's $\eta$-function, Invent. Math. 15 (1972), 91-143.

15. G. Mason, $M_{24}$ and certain automorphic forms, Contemp. Math., vol. 45, Amer. Math. Soc., Providence, RI, 1985, pp. 223-244.

16. M. Newman, Construction and application of a class of modular functions (II), Proc. London Math. Soc. (3) 9 (1959), 373-387.

17. K. Ono, On dihedral representations of modular forms with complex multiplication, preprint.

18. K. Ribet, Galois representations attached to eigenforms of Nebentypus, Lecture Notes in Math., vol. 601, Springer-Verlag, New York, 1977, pp. 17-52.

19. $\ldots$, On l-adic representations attached to modular forms (II), Glasgow J. Math. 27 (1985), 185-194.

20. K. Rosen, Elementary number theory and its application, Addison-Wesley, Reading, MA, 1984.

21. J.-P. Serre, Divisibilité de certaines fonctions arithmétiques, Enseign. Math. (2) 22 (1976), 227-260. 
22. __ Modular forms of weight 1 and Galois representations, Algebraic Number Fields (A. Frolich, ed.), Academic Press, New York, 1977, pp. 193-268.

23. _ـ Quelques applications du théorème de densité de Chebotarev, Inst. Hautes Études Sci. Publ. Math. 54 (1981), 123-201.

24. __ Sur la lacunarité des puissances de $\eta$, Glasgow Math. J. 27 (1985), 203-221.

25. J.-P. Serre and H. Stark, Modular forms of weight $1 / 2$, Lecture Notes in Math., vol. 627, Springer-Verlag, New York, 1977, pp. 29-68.

26. G. Shimura, On modular forms of half-integral weight, Ann. of Math. (2) 97 (1973), 440-481.

27. J. Sturm, Theta series of weight $\frac{3}{2}$, J. Number Theory 14 (1982), 353-361.

28. M. Subbarao and M. Vidyasagar, On Watson's quintuple product identity, Proc. Amer. Math. Soc. 26 (1970).

29. H. P. F. Swinnerton-Dyer, On l-adic representations and congruences for coefficients of modular forms, Lecture Notes in Math., vol. 350, Springer-Verlag, New York, 1973, pp. 1-55.

30. J. Tunnell, $A$ Classical diophantine problem and modular forms of wt. $3 / 2$, Invent. Math. 72 (1983), 323-334.

Department of Mathematics, The University of Georgia, Athens, Georgia 30602 Current address: Department of Mathematics, University of Illinois, Urbana, Illinois 61801

E-mail address: onoesymcom.math.uiuc.edu

Department of Mathematics, University of Northern Colorado, Greeley, Colorado 80639

E-mail address: srobins@dijkstra.univnorthco.edu 